gekleidet wurde und diese somit ebenfalls phosphorhaltiges Roheisen verarbeiten können; gerade der basische Martinofen hat außerordentlich an Gebiet gewonnen. Bemorkt zu werden verdient noch, daB der Thomasprozeß in Deutschland über 2,6 Mill. Tonnen (1908) Thomasschlacke liefert, wovon die Hälfte unserer Landwirtschaft wieder zugute kommen Auch einzelne Martinverfahren liefern jetzt eine der Thomasschlacke fast gleiche phosphorreiche Schlacke.

Da noch mehrere der Pioniere deutscher Thomasarbeit leben, so hat die Redaktion von ,Stahl und Eisen" den sehr glücklichen Gedanken gehabt, diese um die Einführung und Durchbildung des Verfahrens sehr verdienten Herren selbst aus ihrer Erfahrung über die Anfänge der deutschen Thomasflußeisenerzeugung berichten zu Iassen. Diese von Massenez,Hilgenstock, S pannagel, G. $\mathrm{M}$ e y $\mathbf{r}^{{ }^{69}}$ ) entworfenen Schilderungen lassen in sehr fesselnder Weise die anfangs zu überwindenden Schwierigkeiten orkennen. Eine Beschreibung des neuen Thomasstahlwerkes der Burbacher Hütte hat S c h r ö d t e $\mathbf{r}^{60}$ ) gebracht.

Ein Teil des Stahles geht in der Form von $S t a h$ f o r $m g$ u $B$ in die Industrie. Die Herstellung geschieht in kleinen sauren oder basischen Martinöfen, in sog. Kleinkonvertern, in Tiegeln und im elektrischen Ofen. Stroughton ${ }^{61}$ ) gibt eine Ubersicht über diese verschiedenen Verfahren nach Betrieb, Leistung, Kosten. Speziell mit der Praxis der Kleinbessemerei befassen sich mehrere Abhandlungen ${ }^{62}$ ).

Die Elektrostah lerzeugung hat im abgelaufenen Jahre weitere Fortschritte gemacht es sind im ganzen über 100 Öfen in industriellem Betriebe, deren jährliche Leistung im abgelaufenen Jahre rund $50000 \mathrm{t}$ betragen haben wird. Deutschland stellte hierzu $17773 \mathrm{t}$ (eine Angabe, die wahrscheinlich zu niedrig ist).

Eine Reihe Abhandlungen versuchen, den wirklichen Vorteil des Elektrostahlofens gegenüber den anderen hüttenmännischen Raffinationsapparaten festzustellen. Die Uberlegenheit beruht in erster Linie auf der Möglichkeit einer energischen Desoxydation des Stahlbades; diese ist bedingt durch die Abwesenheit einer oxydierenden Atmosphäre; sie gestattet eine ganz außerordentlich weitgehende Entschwefelung, ferner ein längeres Abstehen des Bades zur Ausscheidung von Gasen und Schlackeneinschlüssen und die Gewinnung von genau zusammengesetzten Legierungen. $\mathrm{H}_{\mathrm{o}} \mathrm{w}^{\mathrm{62}}$ ) erörtert diese Verhältnisse genauer, auch $E$ i c h h of f ${ }^{63}$ ) beleuchtet die Frage der Desoxydation eingehend. Úber die Wirkung des Abstehens sind $\mathrm{Ho}$ w e und d e Co u s s r g u e s allerdings verschiedener Ansicht. Die Entschwefelung ist durch eine Diskussion zwischen A m berg, S c h m id, Ne u man n ${ }^{64)}$ genügend geklärt.

59) Stahl u. Eisen 1909, 1465. Ref. in dieser Z. 23,136 (1910).

80) Gießereiztg. 1909, 7, 39, 68

61) GieBereiztg. 1909, 493, 567, 635. Stahl u. Eisen 1909, 470, 1190.

62) Rev. de Metall. 1909, 1138.

63) Verh. Ver. Beförd. d. Gewerbefleiß. 1909, 172.

64) Stahl u. Eisen 1909, 176, 355, 1665. Ref. in dieser Z. 22,745 (1909).
C. de ('ous sergues ${ }^{65}$ ) liefert eine ausführliche Schilderung über eine ganze Reihe von ihm besuchter Ofenanlagen unter Angabe von Leistungen, Arbeitsweise, Raffinationsmethode, Kosten. Allgemeiner gehalten ist eine Betrachtung $\mathrm{S}$ a ille r $\mathbf{s}^{66}$ ) über den Elektrostahl, seine Bedeutung für die Stahlindustrie und seine Stellung zu den übrigen Stahlsorten.

Nebenher ist eine sehr große Zahl von Beschreibungen einzelner Ofensysteme, deren Bauart, Leistung usw. veröffentlicht worden. Die Charakterisierung und Differenzierung der einzelnen Systeme dürfte hier zu weit führen; es soll deshalb der Hinweis auf die betreffende Literatur genügen. Der bisher größte Elektrostahlofen ist auf den SüdChicagowerken in Gang gesetzt worden, es ist ein Héroult scher Ofen von $15 \mathrm{t}$ Fassung, er verarbeitet das aus einem sauren Konverter kommende Flußeisen auf sog. Mittelqualität (Schienen usw.) ${ }^{67}$ ). Die Beschreibung eines kleinen Experimentierofens Héroultschen Systems gab Hansen ${ }^{88}$ ). Keller hat neben seinem dem Héroultschen Ofen gleichen System, wovon ein $8 \mathrm{t}$-Ofen in Unieux arbeitet, einen neuen Ofen mit stets leitender Sohle aus Eisenstäben gebaut ${ }^{69}$ ), von dem ein Exemplar auch jetzt nach Deutschland kommt. Die Konstruktion des G i r o d schen Ofens ist wiederholt beschrieben worden. G i r o d betreibt bereits 12,5 tOfen seines Systems ${ }^{70}$ ). Weitere Mitteilungen betreffen die Offen von $\mathrm{Ch}$ a pelet in Allevard71), von Rochling-Rodenhauser ${ }^{72}$ ), Frick ${ }^{73}$ ) und $S$ ch neider ${ }^{76}$ ).

[A. 171.]

\section{Geheimnisverrat der chemischen Angestellten ${ }^{1}$ ).}

\section{Von Rechtsanwalt A. Axster, Berlin.}

(Eingeg. 14.|5. 1910.)

In den deutschen Landen gab es früher einen Schutz gegen unlauteren Wettbewerb überhaupt nicht. Weder existierten Spezialvorschriften, welche gegen die Auswüchse des unlauteren Wettbewerbs im geschäftlichen Verkehr gerichtet waren, noch auch konnte sich die deutsche Rechtsprechung dazu entschließen, die in sämtlichen Rechtsgebieten existierenden Vorschriften über Schadenersatz auf

65) Rev. de Metall. 1909, 589.

66) Österr. Z. f. Berg- u. Hüttenw. 1909, 775. Ref. in dieser Z. 23, 279 (1910).

67) Electr. u. Met. Ind. 1909, 260. Ref. in dieser Z. 22, 1607 (1909).

68) Electr. u. Met. Ind. 1909, 206. Ref. in dieser Z. 22, 1464 (1909).

69) Stahl u. Eisen 1909, 1302. Electr. u. Met. Ind. $1909,255$.

70) Metallurgie 1909, 673. Stahl u. Eisen 1909, 1760. Ref. in dieser Z. 23, 279 (1910).

71) Rev. de Metall. 1909, 589. Vgl. diese $Z$. 22, 1607 (1909).

72) Stahl u. Eisen 1909, 794.

73) Electr. u. Met. Ind. 1909, 363, 368, 450.

74) Ósterr. Z. f. Berg- u. Hüttenw. 1909, 154.

1) Vortrag gehalten in der Fachgruppe für gewerblichen Rechtsschutz gelegentlich der Hauptversammlung des Vereins deutscher Chemiker zu München am 20./5. 1910. 
das Gebiet des unlauteren Wettbewerbs anzuwenden. Anders stand es damit in Frankreich. Dort wurde durch die Rechtsprechung die bekannte Vorschrift des Art. 1382 des code civil im weitesten Umfang dazu angewendet, um einen Schutz gegen die concurrence deloyale zu gewähren. Jene Vorschrift enthält den Satz, daß jede Handlung eines Menschen, welche einem anderen Schaden verursacht, denjenigen zum Ersatze verpflichtet, durch dessen Verschulden sie eingetreten ist. Auf dem hier interessierenden Gebiete der Bekämpfung des Geheimnisverrates enthält aber auch der code penal in Artikel 418 eine ausdrückliche Vorschrift, welche lautet:

„Jeder Direktor, Kommis, Arbeiter einer Fabrik, welcher Fremden oder in fremden Ländern sich aufhaltenden Franzosen Geheimnisse der Fabrik, bei welcher er angestellt ist, mitteilt, soll mit Einsperrung und einer Geldstrafe von 500 bis 20000 Frs. bestraft werden. Sind solche Geheimnisse Franzosen, die in Frankreich wohnen, mitgeteilt worden, so soll die Geldstrafe in dreimonatlichem bis zweijährigem Gefängnis und in einer Geldbuße von 16 bis 200 Frs. bestehen."

Interessant ist an dieser Vorschrift, wie nebenbei bemerkt sei, die Unterscheidung zwischen Fremden und Franzosen, welche, wie dies auch sonst in der französischen Gesetzgebung vielfach der Fall ist, auf Erwägungen beruht, die dem Schutz der nationalen Arbeit dienen sollen; doch dies nur nebenbei.

Mit der außerordentlichen Entwicklung, welche die Industrie in Deutschland seit der zweiten Hälfte des vorigen Jahrhunderts genommen hat, und welche Deutschland zu einem der orsten Industriestaaten der Welt gemacht hat, wurden auch immer mehr Wünsche laut, welche auf eine Erweiterung des gewerblichen Rechtsschutzes und auf einen Schutz gegen die Auswüchse des unlauteren Wettbewerbs hinausliefen. Da die Judikatur, wie gesagt, in der Anwendung der allgemeinen Vorschriften auf das Gebiet des unlauteren Wettbewerbs völlig versagte, so führten diese Bestrebungen schließlich zu dem Erlaß des Spezialgesetzes gegen unlauteren Wettbewerb vom 27./5. 1896. In diesem Gesetz befindet sich eine ausdrückliche Vorschrift gegen den Geheimnisverrat, welche unverändert in das Gesetz in der neuen Fassung vom 7./6. 1909 übernommen wordon ist. Im alten Gesetz war dies $\$ 9$, jetzt ist es $\$ 17$. Das neue Gesetz hat dann in bezug auf diese Materie ganz neu die Vorschrift des $\S 18$ hinzugefügt, auf welche später ausführlich eingegangen werden soll.

Die Vorschriften über den Geheimnisverrat nnd alles, was damit zusammenhängt, haben eine außerordentliche Bedeutung gerade für das Gebiet der Chemie, da es auf diesem Gebiete zahlreiche Betriebsgeheimnisse, technische Vorschriften und Rezepte gibt, welche sich auf chemische Zusammensetzungen beziehen, und an deren Geheimhaltung der Geschäftsherr ein großes und weitgehendes Interesse hat. Diesem Interesse des Geschäftsherrn an der Geheimhaltung stehen auf der anderen Seite die erhoblichen Nachteile entgegen, welche den Angestellten für ihr Fortkommen aus der Uberspannung des Prinzips der Geheimhaltungspflicht erwachsen können. Wie das Gesetz sich zu diesen beiderseitigen Interessen stellt, welche Rechte es dem Geschäftsherrn verleiht, und welche Pflichten es den Angesteliten auferlegt, dies zu untersuchen, ist unsere Aufgabe. Die Bestimmungen des Gesetzes vom 7./6. 1909, welche hier in Betracht kommen, sind folgende:

\section{$\$ 17$.}

,Mit Gefängnis bis zu 1 Jahre und mit Geldstrafe bis zu $5000 \mathrm{M}$ oder mit einer dieser Strafen wird bestraft, wer als Angestellter, Arbeiter oder Lehrling eines Geschäftsbetriebes Geschäfts- oder Betriebsgeheimnisse, die $\mathrm{ihm}$ vermöge des Dienstverhältnisses anvertraut odor sonst zugängig geworden sind, während der Geltungsdauer des Dienstverhältnisses unbefugt an andere zu Zwecken des Wettbewerbs oder in der Absicht, dem Inhaber des Geschäftsbetriebes Schaden zuzufügen, mitteilt. Gleiche Strafe trifft denjenigen, welcher Geschäftsoder Betriebsgeheimnisse, deren Kenntnis er durch eine im Absatz 1 bezeichnete Mitteilung oder durch eine gegen das Gesetz oder die guten Sitten verstoßende eigene Handlung erlangt hat, zu Zwecken des Wettbewerbs unbefugt verwertet oder an andere mitteilt."

\section{$\S 18$.}

„,Mit Gefängnis bis zu I Jahre und mit Geldstrafe bis zu $5000 \mathrm{M}$ oder einer dieser Strafen wird bestraft, wer die ihm im geschäftlichen Verkehr anvertrauten Vorlagen oder Vorschriften technischer Art, insbesondere Zeichnungen, Modelle, Schablonen, Schnitte, Rezepte zu Zwecken des Wettbewerbs unbefugt rerwertet oder an andere mitteilt."

\section{$\S 19$.}

"Zuwiderhandlungen gegen die Vorschriften der $\$ 17$ und 18 verpflichteten außerdem zum Ersatz des entstandenen Schadens. Mehrere Verpflichteten haften als Gesamtschuldner."

In Betracht kommt außerdem noch \$ 1, welcher lautet:

„Wer im geschäftlichen Verkehr zu Zwecken des Wettbewerbs Handlungen vornimmt, die gegen die guten Sitten verstoßen, kann auf Unterlassung und Schadenersatz in Anspruch genommen werden."

Es ist nun unsere Aufgabe, diese Bestimmungen im einzelnen kurz zu erläutern, und zwar sowohl in strafrechtlicher, als auch in zivilrechtlicher Hinsicht, und des ferneren das Verhältnis der Einzelbestimmungen zueinander zu beleuchten. Täter der strafbaren Handlung aus $\$ 17$ des Gesetzes sind Angestellte, Arbeiter oder Lehrlinge eines Geschäftsbetriebes. Auf die umstrittene Frago, ob das Gesetz die Arbeiter und Lehrlinge den Angestellten hat koordinieren wollen, oder "Angestellte" der allgemeino Begriff sein soll, unter welchen auch die Arbeiter und Lehrlinge fallen sollen, einzugehen, ist überflüssig, da praktisch beides genau auf dasselbe hinausläuft, und hier nicht der Ort dazu ist, um juristische Doktorfragen zu entscheiden, vielmehr lediglich auf die Bedeutung der Vorschriften für die Praxis ankommt. Praktisch kann eben Täter jeder Angestellte sein. Fs muß sich um Angestellte eines Geschäftsbetriebes handeln. Der Begriff Geschäftsbetrieb umfaßt alle Gebiete des gewerblichen Lebens, für das Gebiet der Chemie also meines Erachtens wohl alle denkbaren Betriebe, soweit dieselben irgendwie einen gewerblichen Charakter haben. Ein 
wissenschaftliches Laboratorium einer Lehranstalt gehört dagegen zu einem Geschäftsbetriebe nicht.

Gegenstand der strafbaren Handlung ist der Verrat von Geschäfts- und Betriebsgeheimnissen des Geschäftsinhabers. Fs wird nun in erster Reihe zu untersuchen sein, was unter einem Geheimnis im Sinne der gesetzlichen Bestimmungen zu verstehen ist. Das Gesetz selbst gibt eine Definition des Begriffs Geheimnis nicht. Man hat eine solche absichtlich vermieden, und zwar wegen der Schwierigkeit einer allseitig ausreichenden Bestimmung der einzeinen Merkmale.

Unter Geheimnis ist zunächst, wie das Wort ohne weiteres ergibt, eine Tatsache zu verstehen, welche nicht offenkundig ist. Offenkundig ist eine Tatsache, welche einer unbegrenzten Anzahl von Porsonen zugänglich oder ihnen bekannt ist. Der Schwerpunkt liegt in dem Wort unbegrenzt. Die Kenntnis einer größeren Anzahl von Personen von einer Tatsache, weiche aber anderen Personen nicht bekannt und zugängig ist, schließt an sich den Begriff des Geheimnisses noch nicht aus. Als Geheimnis im Sinne des vorliegenden Gesetzes kann allerdings nur eine solche Tatsache angesehen werden, von welcher nur solche Personen Kenntnis haben, welche ein Recht an dem Geheimnis haben. Praktisch ausgedrückt bedeutet dies, da日 im Geschäftsbetriebe Geheimnisse sich auf solche Tatsachen beziehen, welche dem Geschäftsinhaber oder mehreren Geschäftsinhabern oder Vertretern der Geschäftsinhaber als solchen bekannt geworden sind und anderen Personen mit Willen der Geschäftsinhaber nicht oder nur mit der Auflage der Geheimnisbewahrung bekannt geworden sind.

Selbstverständlich muß die das Geheimnis bildende Tatsache dem Empfänger der Mitteilung unbekannt sein, da eine noch so geheime Tatsache, wenn sie zufällig gerade demjenigen, dem sie mitgeteilt wird, bekannt ist, für diesen eben kein Geheimnis ist, von einem Geheimnisverrat also nicht die Rede sein kann.

Die in Betracht kommende Tatsache muß o b jekti v ein Geheimnis sein, d. h. sie maß an sich diejenigen . Bedingungen erfüllen, welohe vorstehend für den Begriff des Geheimnisses aufgestellt sind. Eine an sich allgemein bekannte Tatsache kann nicht dadurch zum Geheimnis im Sinne der hier in Frage stehenden Bestimmungen worden, daß der Geschäftsinhaber dem Angestellten vertraglich zar Pflicht macht, dieselbe anderweitig nicht mitzuteilen. Die praktische Folge ist die, daB die Mitteilung einer solchen an sich bekannten Tatsache, deren Mitteilung aber dem Angestellten von dem Geschäftsherrn untersagt ist, niemals a t $\mathrm{r}$ a $\mathrm{f}$ b a r sein kann. Zivilrechtlich könnte allerdings wohl der Geschäftsherr auf Grund allgemeinrechtlicher Grundsätze die Unterlassung der Mitteilung solcher Tatsachen erzwingen und wegen trotzdem erfolgter Mitteilung ev. auch Schadenersatz verlangen. Auf Grund der Bestimmungen des vorliegenden Gesetzes könnte er dies aber jedenfalls nicht, da dieses immer ein objektives Geheimnis im Sinne der vorstehenden Ausführung voraussetzt.

Kann nun jederlei Tatsache ein Geheimnis darstellen, dessen Verrat strafbar ist, oder muß hier ein gewisser qualitativer Unterschied gemacht wer- den? lch nehme das letztere an. Eine Ausartung zur Geheimniskrämerei ist nicht angängig. Dio betreffende Tatsache muß für den Geschäftsbetrieb von irgendwelcher Bedeutung sein. Vorschriften und Rezepte ganz untergeordneter gleichgültiger Natur, welche der Geschäftsherr aus ingendwelchen Gründen vielleicht zu Geheimnissen zu stempeln versucht, müssen meines Erachtens auBer Betracht bleiben, so daB also in jedem Falle zu prïfen sein wird, ob dem betreffenden sog. Geheimnis irgendwelche Bedeutung innewohnt.

Von großer Wichtigkeit ist die Frage, ob dasjenige, was geheim zu halten ist, $n$ e $u$ sein muß, Für den Begriff der Neuheit wird man das zugrunde zu legen haben, was andere den gewerblichen Rechtsschutz betreffende Gesetze in dieser Hinsicht als Voraussetzungen aufstellen. Neu ist hiernach ein Gegenstand - Gegenstand im weitesten Sinne gebraucht - dann nicht, wenn er früher einmal offenkundig benutzt oder in öffentlichen Druckschriften beschrieben worden ist. Diese Vorsussetzung ist für den Begriff des Geheimnisses, wie übrigens auch das Reichsgericht entschieden hat, nicht aufzustellen. Es kann also sehr wohl z. B. ein Verfahren, welches früher einmal offenkundig benutzt oder öffentlieh beschrieben worden ist, ein Geheimnis sein, wenn nur objektiv festgestellt werden kann, $\operatorname{da} \beta$ zu der in Betracht kommenden Zeit die allgemeine Kenntnis bzw. der offenkundige Gebranch nicht mehr vorhanden ist, und wenn die Druokschriften den interessierten Kreisen nicht mehr bekannt oder gar schon verschollen sind. Zu welchen Konsequenzen die gegenteiligen Bestimmungen insbesondere des Patent- und Gebrauchsmustergesetzes führen, hat schon mancher am eigenen Leibe orfahren müssen. Werden doch so und so oft von solchen Leuten, welche den Gegenstand eines fremden Patentes in Gebrauch nehmen wollen, alte verschollene und vergilbte Druckschriften, von deren Vorhandensein kein Mensch mehr eine Ahnung hatte, mit vieler Müho hersusgesucht, um ein wertvolles Patent zu Falle zu bringen, und um die Früchte fremder Tätigkeit und fremder Geistesarbeit sich zunntze zu machen. Doch diese Bemerkung nur nebenbei, da sio ja nicht zu dem eigentlichen Thema gehört.

Fs ist bereits vorher ausgeführt worden, daß der Geheimhaltungswille des Geschäftsherrn bei solchen Tatsachen, welche objektiv kein Geheimnis sind, nicht ausreicht, um sie zum Geheimnis zu stempeln; andererseits reicht es aber natïrlich auch nicht aus, daß die betreffende Tatsache objektiv ein Geheimnis ist. Es muß vielmehr immer noch der Geheimhaltungswille des Besitzers des Geheimnisses hinzukommen. Eigentlich ist dies ja selbstverständlich. Wenn der Geschäftsherr eine Tatsache, die ihm allein bekannt ist, und die auch die Eigenschaft eines Geheimnisses besitzen wïrde, einem Angestellten mitteilt und $\mathrm{ihm}$ dabei ausdrücklich oder stillschweigend zu erkennen gibt, daß ihm an der Geheimhaltung gar nichts gelegen ist, so bleibt diese Tatsache natürlich nicht ein Geheimnis im Sinne des Gesetzes, und ihre weitere Mittoilung kann niemals als Geheimnisverrat angesehen werden. Es muß aber, wie gesagt, ausdrücklich oder stillschweigend zam Ausdruck gebracht sein, daB dem Geheimnisbesitzer an der Geheimhaltung nichts 
gelegen ist. Allein daraus, daB die Geheimhaltung nicht ausdrücklich auferlegt ist, kann man noch nicht folgern, daß eine solche auch nicht notwendig ist, vielmehr wird man bei wirklichen Geheimnissen auch immer einen Geheimhaltungswillen vermuten müssen. Der Angestellte, welcher eine Tatsache, die Gegenstand eines Geheimnisses ist, weiter gegeben hat, kann sich also zu seiner Entsehuldigung nicht lediglich darauf berufen, daß ihm ja nicht ausdrücklich verboten worden sei, diese Tatsacho anderen mitzuteilen. Er wird vielmehr, wenn es sich wirklich um ein Geheimnis handelt, darzutun haben, daß nach ausdrücklichen Erklärungen oder nach Lage der Sache er nicht annehmen konnte, daß der Geschäftsherr diese Tatsache habe geheimhalten wollen.

Soviel über den Begriff des Geheimnisses.

Es muß nun weiter ein Ges chäfts - oder $B$ e tri e b s geheimnis vorliegen. Unter Geschäftsgeheimnissen wird man mehr solche kaufmännischer Art, unter Betriebsgeheimnissen solche technischer Art zu verstehen haben. Praktisch ist dieser Unterschied nicht von großer Bedeutung. Geschäftsgeheimnisse werden hauptsächlich für kaufmännische Angestellte in Frage kommen, so daß ein Eingehen auf dieselben sich im Rahmen der gegenwärtigen Erörterung wohl erübrigt. Betriebsgeheimnisse kommen für technische Angestellte in Frage, interessieren also hier eigentlich ausschließlich. Betriebsgeheimnis ist ein solches, welches mit dem Betriebe des Geschäftsherrn in irgendwelcher Beziehung steht. Ein reines Privatgeheimnis ist daher kein Betriebsgeheimnis. Es ergibt sich dies ja ohne weiteres schon aus der ganzen Tendenz des Gesetzes, welches den unlauteren Wettbewerb im geschäftlichen Verkehr bekämpfen will. Beispiele für Betriebsgeheimnisse brauche ich wohl nicht anzuführen, da solche den Herren vermutlich in weit größerem Umfange bekannt sein werden, als mir.

Geheimnisverrat liegt nur dann vor, wenn das betreffende Geheimnis dem Angestellten vermöge seines Dienstverhältnisses anvertraut oder sonst zugängig gemacht worden ist. Das Gesetz hätte sich mit den Worten ,zugängig gemacht worden ist" wohl begnügen können, da das Anvertrauen nur ein Unterbegriff des Zugängigmachens ist. Die Kenntnis muß vermöge des Dienstverhältnisses erlangt sein. Damit ist aber nicht gesagt, da $B$ es sich nur um Geheimnisse handeln muß, die dem Thter in Verrichtung der ihm zugewiesenen Beschäftigung bekannt geworden sind. Der Begriff ist vielmehr ein weiterer. Es genügt schon, wenn dem Täter die betreffende Tatsache nur aus Anlaß seines Dienstverhältnisses zur Kenntnis gekommen ist. Der Kommentar von R o senthal-Wehner zum Gesetz gegen den unlauteren Wettbewerb, auf dessen klare und übersichtliche Darlegungen ich mich überhaupt vielfach stütze, gibt folgendes Beispiel:

„Ein in einem Geschäft angestellter Kontordiener, welchem in dieser Eigenschaft die. Schlüssel zum Privatkontor des Geschäftsherrn zur Verfügung stehen, schließt dasselbe auf und liest die darin befindlichen Geheimrezepte durch, um sie einem dritten zu verraten."

Hier ist, wenn auch die dienstlichen Verrichtungen des Kontordieners mit den betreffenden Ge- heimrezepten nicht das mindeste zu tun haben, zweifellos anzunehmen, daB der Kontordiener von dem Geheimnis vermöge seines Dienstverhältnisses Kenntnis erlangt hat.

Im Anschluß hieran möchte ich noch auf die, gerade für die chemischen Angestellten meines Erachtens sehr wichtige Frage eingehen, ob ein Angestellter, welcher innerhalb seines Tätigkeitsfeldes selbst ein neues Rezept oder eine Vorschrift für eine Zusammensetzung gefunden hat, seinerseita dadurch, daß er dies Rezept an dritte weitergibt, einen Geheimnisverrat begehen kann. Daß derartige Rezepte oder Vorschriften oder Erfindungen an sich dem Geschäftsherrn zufallen, daß ihm also die Ausnutzung derselben zusteht, wird man nach Lage unserer Rechtsprechung kaum in Zweifel ziehen können. Kann man nun aber bei dem Angestellten, welcher die Erfindung selbst gemacht hat, davon reden, daß ihm dieselbe anvertraut oder vermöge seines Diensverhältnisses zugänglich geworden sei? Von einem Anvertrauen kann natürlich nicht die Bedo sein. Wie steht es nun aber mit der Frage, ob dem Angestellten die Erfindung in diesem Falle vermöge seines Diensverhältnisses zugänglich geworden ist. Hierbei muß man erwägen, ob der Angestellte die betreffende Erfindung - ich spreche nur beispielsweise von einer Frfindung - in Erfüllung seiner Dienstpflichten and innerhalb des ihm zugewiesenen Geschäftskreises gemacht hat. Wenn, wie bereits gesagt, die betreffende Erfindung an sich dem Geschäftsherrn zufällt, so ist ohne weiteres ersichtlich, daß bei hinzukommendem Geheimhaltungswillen des Geschäftsherrn die betreffende Erfindung zum Betriebsgeheimnis wird. $\mathrm{Ob}$ man nun weiter schließen kann, daß nunmehr die zu einem Betriebsgeheimnis gewordene Erfindung dem Angestellten, welcher die Erfindung ursprünglich selbst gemacht hat, wiederum vermöge seines Dienstverhältnisses zugänglich wird, ist mir zweifelhaft. Etwas gesucht ist diese Konstruktion jedenfalls, Ich habe erhebliche $Z_{w}$ weifel, ob der betreffende Angestellte, wenn er das Geheimnis anderen mitteilt, den Strafbestimmungen des Gesetzes unterliegt. Denn eigentlich sagt doch der gesunde Menschenverstand, daß jemandem etwas, was er selbst erfunden hat, nicht hinterher vermöge seines Dienstverhältnisses zugänglich werden kann. Trotzdem wird die gegenteilige Konsequenz von Kommentatoren und anscheinend auch in der Rechtsprechung teilweise gezogen. Natïrlich hat hiermit die Frage, ob nicht auch die Wahrung solcher Geheimnisse Angestellten im Vertragswege auferlegt werden kann, nichts zu tun. Nur ziehen Vertragsverletzungen keine strafrechtliche Ahndung nach sich.

Die Mitteilung des Geschäfts- und Betriebsgeheimnisses muß dann weiter unbefugt und vorsätalich erfolgen. Unbefugt ist eine Mitteilung dann, wenn der Geschäftsherr weder ausdrücklich noch stillschweigend seine Zustimmung zu der Mitteilung erteilt hat. Eine stillschweigende Zustimmung ist dann anzunehmen, wenn aus irgendwelchen Handlungen des Geschäftsherrn zu entnehmen ist, daß er gegen die Mitteilung nichts einzuwenden habe. Unter Vorsätzlichkeit versteht man das Bewußtsein des Angestellten, daß er anderen ein Geheimnis unbefugt zu Zwecken des Wettbewerbs mitteilt. Fahrlässigkeit ist nicht 
strafbar. Wenn also die Sachlage ergibt, daß der Angestellte, wenn auch aus unentschuldbarem Irrtum geglaubt hat, daß er zur Mitteilung der betreffenden Tatsache befugt sei, so kann er wegen dieser Mitteilung nicht bestraft werden. Es kann dies z. B. der Fall sein, wenn der Angestellte eine Äußerung des Geschäftsherrn mißverstanden hat und auf Grund dieses Mißverständnisses zu der Auffassung gelangt ist, daß er diese Tatsache ruhig weiter mitteilen dürfe.

Die Mitteilung muß, um strafbar zu sein, zu Zwecken des Wettbewerbs oder in der Absicht, dem Geschäftsherrn Schacien zuzufügen, geschehen sein. Ich glaube nicht, daß es hier am Platze ist, juristische Darlegungen darüber zu machen, was unter den Worten ,,zu Zwecken des Wettbewerbs" zu verstehen ist. Das Reichsgericht hat einmal gesagt, daß der Wettbewerb in der Heranziehung von Kunden bzw. der Zurückdrängung eines Miterwerbers im eigenen oder fremden Interesse besteht. Die Mitteilung muß zu Zwecken des Wettbewerbes erfolgen, d. h., der Mitteilende muß die Absicht haben, durch die Mitteilung seinem oder fremdem Wettbewerb zu dienen. Was unter der Absicht, dem Geschäftsherrn Schaden zuzufügen, zu verstehen ist, bedarf wohl keiner Erläuterung.

Der Schutz des $\$ 17$ beschränkt sich auf die Geltungsdauer des Dienstverhältnisses. Der Angestellte ist also nach \$ 17 nur strafbar, wenn er das betreffende Geheimnis während derjenigen Zeit an einen anderen verrät, während welcher er bei dem Geschäftsherrn tatsächlich noch in Stellung ist.

Die Strafen für Geheimnisverrat der Angestellten sind Gefängnis bis zu einem Jahre und zugleich Geldstrafe bis zu 5000 M oder beide Strafen wahlweise.

Absatz 2 des $\$ 17$ interessiert hier im wesentlichen nicht, $\mathrm{da}$ er in erster Linie denjenigen treffen soll, welcher das Geheimnis von einem Angestellten erfahren hat und es seinerseits verwertet. Ganz auszuschalten ist aber der Absatz 2 dennoch nicht, da or auch denjenigen unter Strafe stellt, welcher Betriebs- und Geschäftsgeheimnisse, deren Kenntnis er durch eine gegen das Gesetz oder die guten Sitten verstoßende eigene Handlung erlangt hat, zu Zwecken des Wettbewerbs unbefugt verwertet oder an andere mitteilt. Absatz 2 des $\$ 17$ kann sich daher sehr wohl auch auf Angestellte beziehen. Denn nach dieser Vorschrift ist ein Angestellter, welcher ein Geheimnis, dessen Kenntnis er durch eine gegen das Gesetz oder die guten Sitten verstoßende eigene Handlung erlangt hat, verwertet oder mitteilt, strafbar. Viel Raum dürfte für die Anwendung dieser Bestimmung neben dem Abs. 1 des $\$ 17$ allerdings kaum gegeben sein. Es könnte z. B. ein Angestellter eines Betriebes des Nachts in das verschlossene Privatkontor des Geschäftsherrn einen Einbruch verüben und dabei Geheimrezepte stehlen, welche er dann weitergibt. Auch dieser Angestellte würde natürlich, abgesehen von der Strafe des Ginbruchsdiebstahls, wegen Geheimnisverrats strafbar sein. Diese Strafbarkeit hätte aber mit dem Angestelltenverhältnis nichts zu tun, da sie jeden anderen, der das Gleiche täte, auch träfe. Infolgedessen kann die Bestimmung des $\mathrm{Ab}$ satzes 2 bei der vorliegenden Erörterung wohl ausscheiden.
Die Beschränkung der Strafbarkeit aus $\$ 17$ Abs. 1 des Gesetzes auf die Dauer des Vertragsverhältnisses leitet uns auf $\$ 18$ des Gesetzes über, welcher eine solche Beschränkung nicht enthält. Die Vorschrift des $\$ 18$ in dem Gesetze vom 7./6. 1909 ist eine ganz neue. Sie ist in das Gesetz hineingekommen auf eine Anregung aus den Kreisen der Stickereiund Spitzenindustrie, welche Klage darüber geführt hatte, daß von Unternehmern mit den ihnen von den Fabrikanten zur Ausführung von Aufträgen übergebenen Schablonen Mißbrauch getrieben werde. Die Bestimmung hat dann aber schließlich eine viel weitergehende Bedeutung erhaiten, indem ganz allgemein die unbefugte Mitteilung von im geschäftlichen Verkehr anvertrauten Vorschriften technischer Art unter Strafe gestellt ist. Als Beispiel der Vorschriften technischer Art werden auch Rezepte angeführt. Diese Vorschrift hat also eine eminente Bedeutung auch für die chemische Industrie. Unter Vorschriften technischer Art, wozu die Rezepte zu zählen sind, hat man natürlich nur solche Vorschriften zu verstehen, welche ein Verfahren zur Herstellung von gewerblichen Erzeugnissen betreffen. Rein kaufmännische Vorschriften scheiden selbstverständlich aus. Eine Erörterung der übrigen Tatbestandsmerkmale des § 18 insbesondere der Begrifie ,Geschäftsverkehr " „anvertraut", , zu Zwecken des Wettbewerbs" und ,, unbefugt" bedarf es nicht, da sich diese Bezeichnungen mit den betreffenden Bezeichnungen des $\$ 17$ decken. Sehr wichtig ist aber die Frage des Verhältnisses des $\$ 18$ zu dem $\$ 17$. $\$ 17$ bezieht sich nur auf Angestellte, während \$18 ganz allgemein jede Person, also auch nicht Angestellte betrifft. Soweit Angestellte in Frage kommen, liegt, abgesehen von der noch zu erörternden Dauer des Schutzes, der Unterschied nur darin, daß $\S 17$ von dem Verrat anvertrauter oder anderweitig zugängig gewordener Geschäftsgeheimnisse, \$18 aber von dem Verrat anvertrauter Vorschriften technischer Art, also von einer besonderen Gruppe von Betriebsgeheimnissen spricht.

Der Schutz des $\$ 18$ ist, wie gesagt, zeitlich ein weitergehender und beschränkt sich gerade bei Angestellten nicht auf die Dauer des Dienstverhältnisses. Man hat allerdings aus den Worten ,im geschäftlichen Verkehr anvertrauten Vorlagen oder Vorschriften " den Schluß ziehen wollen, daB Voraussetzung der Anwendung des $\$ 18$ das Bestehen eines Vertragsverhältnisses zwischen dem Täter und demjenigen, gegen den der Verrat begangen wird, sei. Man hat daraus weiter gefolgert, daß mit dem Aufhören des Vertragsverhältnisses auch der Schutz des $\$ 18$ zu Ende sei. Wäre diese Ansicht, welche insbesondere in dem Kommentar von Rosent hal-Wehner vertreten wird, richtig, so müßte ich zu dem Schlusse kommen, daB für die chemischen Angestellten die Vorschriften der $\$ 18$ und 17 eigentlich völlig identisch sind. Ich kann diese Ansicht aber nicht als richtig anerkennen. Im Wortlaut des Gesetzes findet sio keinerlei Stütze. Denn es können Personen sehr wohl im geschäftlichen Verkehr zueinander stehen, ohne daß ein Vertragsverhältnis zwischen ihnen besteht. Der Begriff des geschäftlichen Verkehrs ist ein viel weiterer, als der des Vertragsverhältnisses. Beispielsweise soll nur auf die mißbräuchliche $\mathrm{Be}$ nutzung von Ansichtssendungen verwiesen werden. 
Wer einem anderen eine Ansichtssendung zugehen läßt, macht diesem ein Vertragsangebot. Ein Vertrag kommat erst dadurch zustande, daß der Empfänger der Ansichtssendung auf Grund derselben eine Bestellung macht. Tut er dies nicht, so liegt zweifellos ein Vertragsverhältnis nicht vor. Trotzdem kann kein $Z$ weifel darüber bestehen, daß die betreffenden Ansichtssendungen dem Kunden im geschäftlichen Verkehr anvertraut sind, und $\mathrm{da} \beta$ daher sehr wohl durch MiBbrauch des Inhalts der Ansichtssendungen ein Geheimnisverrat begangen werden kann. Auf die Frage, ob die von R ose $\mathbf{n}$ t h a l - W e h n er vertretene Ansicht sich aus der Entstehungsgeschichte des Gesetzes begründen läßt, einzugehen, ist hier nicht der Ort. Wen diese Frage besonders interessiert, der möge den Aufsatz von Rechtsanwalt Wertheimer in Nr. 3 des neunten Jahrgangs der Zeitschrift ,Markenschutz und Wettbewerb" nachlesen. Beschränkt sich aber das Anwendungsgebiet des $\$ 18$ nicht auf Vertragsverhältnisse, so fällt natürlich damit auch die Schlußfolgerung, daß mit dem Aufhören des Vertragsverhältnisses auch der Schutz des § 18 aufhört. Man wird slso snnehmen müssen, $\mathrm{da} B$ der $\$ 18$ gegenüber Angestellten auch über die Dauer ihres Dienstverhältnisses hinaus Anwendung findet. Soll dies nun aber unbeschränkt und bis in alle Ewigkeit der Fall sein? Eine solche Auslegung würde für die Angestellten ganz unübersehbare nachteilige Folgen haben. Man muß also aus dem Gesetz heraus, welches a usdrückli ch hierüber nichts besagt, eine Begrenzung zu finden suchen. Diese Begrenzung liegt, wie $W$ e r t h e i m e $r$ in seinem vorerwähnten Aufsatz meines Erachtens zutreffend ausführt, in den Worten „zu Zwecken des Wettbewerbs.“ Fällt die Möglichkeit eines Wettbewerbs weg, dann erreicht auch das Verbot des $\$ 18$ sein Ende. Fehlt also jedes verständige Interesse des Anvertrauenden an der Geheimhaltung, da ein Wettbewerb gegen ihn nicht oder nicht mehr möglich ist, so entfällt auch der Schutz des Gesetzes. Man denke an den Fall, daß der Anvertrauende sein Geschäft oder den betreffenden Geschäftszweig aufgegeben hat. Es ist allerdings zuv.ugeben, daß sich. hier geranle für den Angestellten oft sehr mißliche Konsequenzen ergehen werden, da es in den meisten Fällen sehr schwer sein wird, das Ende des Schutzes festzustellen, und da damit den Angestellten oft eine unieidliche Beschränkung auf ganz unboschränkte Dauer auferlegt werden kann. Fine andere Auslegung des Gesetze ist meines Frachtens nach aber nun einmal nicht möglich, und es kann daher hier, wenn sich, wie vorauszusehen ist, erhebliche Mißstände erSeben werden, nur auf den Weg der Gesetzesänderung verwiesen werden. $\mathrm{Ob}$ das Reichsgericht etwa, wie schon in manchen Fällen, auch hier in irgend einer Weise auf Grund der bestehenden Gesetzgebung helfen wird, läßt sich jetzt nicht voraussehen.

Die bisherige Erörterung hat sich im wesentlichen auf den strafrechtlichen Schutz gegen den Geheimnisvorrat bezogen. In zivilrechtlicher Boziehung bestimmt $\S 19$, daß Zuwiderhandlungen gegen die Vorschriften der $\$ 17$ und 18 außerdem zum Ersatze des entstandenen Schadens verpflichten. Der Angestellte, welcher sich des Geheimnisverrats nach $\$ 17$ und 18 schuldig macht, muß also dem Geschäftsherrn allen ihm hierdurch erwachsenen Schaden erstatten. Daß bei unseren deutschen Gerichten die rechnungsmäßige Feststellang eines solchen Schadens oft auf große Schwierigkeiten stößt, dürfte allseitig bekannt sein. Daß dem Geschäftsherrn neben dem Schadenersatzanspruche auch ein Anspruch auf Unterlassung weiterer einen Geheimnisverrat darstellender Handlungen zusteht, ist im Gesetz zwar nicht ausdrücklich erwähnt, jedoch ergibt sich dies ohne weiteres aus allgemeinen Rechtsgrundsätzen. Es ist dies auch vom Reichsgericht stets angenommen worden. Ausdrücklich erwähnt ist dieser sog. Unterlassungsanspruch in \$ 1 des Gesetzes vom 7./6. 1909, welcher, wie bereits eingangs gesagt ist, ebenfalls für die zivilrechtliche Seite der Geheimnisverrats in Betracht kom. men kann. Wenn § 1 nämlich bestimmt, daß derjenige auf Unterlassung und Schadenersatz in Anspruch genommen werden kann, welcher im geschäftlichen Verkehr zu Zwecken des Wettbewerbs Handlungen vornimmt, die gegen die guten Sitten verstoßen, so sind darin eigentlich alle Fälle des unlauteren Wettbewerbs, soweit es sich urm deren zivilrechtliche Verfolgung handelt, mit inbegriffen, also auch der Geheimnisverrat. Denn nach der Auslegung, welche das Reichsgericht in vielfachen Fällen dem Begriff der guten Sitten gegeben hat, im übrigen auch nach gesundem Menschenverstand, wird man wohl annehmen müssen, daß derjenige Angestellte, welcher ein ihm anvertrantes Betriebsgeheimnis zu Zwecken des Wettbewerbs unbefugt an andere mitteilt, eine Handlung begeht, welche gegen die guten Sitten verstößt.

Ich glaube, im wesentlichen diejenigen Punkte berüht zu haben, welche in bezug auf den Geheimnisverrat für chemische Angestellte von Bedeutung und wissenswert sind. Wis die Rechtsprechung diese Vorsohriften auslegt und weiter entwickeln wird, ist zurzeit noch nicht abzusehen. Wir wollen wünsohen, daB dies in einer Weise geschehen wird, welche die bereohtigten Interessen der Geschäftsherren and der Angestellten in verstïndiger Weise auszugleichen verstehen wird.

[A. 149.]

\section{Über quantitative Spektralanalyse.}

\section{Von \\ Walther Hemper u. Ralph L. von Kremperer.}

\section{(Eingeg. 4./7. 1910,}

Während die Absorptionsespektren vielfach für quantitative Bestimmungen benutzt werden, ist bis jetzt keine Methode bekannt, mit der man Emissionsflammenspektren für quantitative Untersuchungen verwenden könnte. Der Grund liegt in der Schwierigkeit, in Flammen genau bestimmte Quantitäten von Stoffen zur Verdampfung zu bringen. $B$ un $\mathrm{s} \in \mathrm{n}$ und $\mathrm{K}$ i r $\mathrm{c}$ h h of führten zum Zwecke spektralanalytischer Beobachtungen die Substanz einfach in der Ose eines Platindrahtes in die Flamme; sie haben, um eine Natriumflamme zu erhalten, der Schwefelsüuro ihres Apparates Kochsalz beigemischt, welches bei der Entwicklung des Wasserstoffes mit Zink in Form kleiner Flüssigkeitsbläschen mitgerissen wird. 